\title{
Applications of inexact programming methods to waste management under uncertainty: current status and future directions
}

\author{
Wei Sun ${ }^{1 *}$, Chunjiang $A n^{1}$, Gongchen $\mathrm{Li}^{1}$ and Ying LV ${ }^{2}$
}

\begin{abstract}
Waste management problems are subject to uncertainties presented as intervals, random variables and/or fuzzy sets. During the past 20 years, inexact programming methods have been developed and applied increasingly to waste management problems under uncertainty. To obtain a snapshot of these studies, this paper gives a review on recent developments, applications, challenges, and barriers associated with inexact programming techniques in supporting waste management. The results indicate that the majority of inexact programming methods can be categorized as two-stage stochastic programming, chance-constrained programming, fuzzy flexible programming, fuzzy robust programming, interval-parameter programming, mixed-integer programming, multiple-objective programming, and nonlinear programming. The demanding areas for future research efforts would include: expansion of conventional concepts to quantify uncertainties, integration of single inexact programming method with other programming methods to deal with multiple uncertainties and even complexities (e.g. nonlinearities and interactions), integration of inexact programming with other modeling techniques (e.g. life cycle assessment, multiple-criteria decision analyses, and waste flow simulation) to support sustainable waste management, development of more efficient algorithms to solve the proposed methods, linkage of waste management with its environmental impacts (e.g. air pollutants and GHG emissions as well as leachate pollution) within an inexact optimization framework, and applications of the developed methods to novel (e.g. specific types of wastes) or real-world waste management cases in different countries.
\end{abstract}

Keywords: Waste management; Interval-parameter programming; Stochastic programming; Fuzzy programming; Uncertainty; Climate change; Review

\section{Introduction}

In a waste management system, there are usually various types of waste-management facilities (e.g., incinerators, landfills, composting plants and recycling centers) with different functions, which are interrelated to each other through the transferred waste flows (Belien et al. 2014; Ghiani et al. 2014). A typical waste management problem is to deal with various components of the waste management system in an economically and environmentally sound manner (Chang et al. 2011). The tradeoff among environmental, economic, and social requirements in waste management brings about challenges for finding

\footnotetext{
* Correspondence: sunwei@iseis.org

${ }^{1}$ Institute for Energy, Environment and Sustainable Communities, University of Regina, Regina, SK S4S 7H9, Canada

Full list of author information is available at the end of the article
}

such cost-effective strategies. Thus, it is indispensable for decision makers to consider different waste-management options using a systems analysis approach (Marshall and Farahbakhsh 2013; Verderame et al. 2010), which can help to get insights among interacted components and capture the essential features of real-world waste management systems (Pires et al. 2011; Juul et al. 2013). Nevertheless, due to availability and quantifiability of related information, the inevitable uncertainties in many system parameters, decision variables, objective functions, and their relationships could make the waste management systems more complicated (Abichou et al. 2010). Therefore, inexact programming methods would be an ideal systems analysis tool to support decisions for various waste management problems. 
During the past several decades, various inexact programming methods were applied to waste management; they were mainly categorized as stochastic mathematical programming (SMP), fuzzy mathematical programming (FMP), interval-parameter mathematical programming (IMP), and combinations of these methods (Singh 2012). These inexact methods can deal with uncertainties expressed in random variables, fuzzy sets, and a single form of interval, as well as more complex forms of uncertainties (Zeng et al. 2011). Among them, the SMP focuses on mathematical programming problems where coefficients in constraints or the objective function are not deterministic but can be quantified as chances or probabilities. Two main types of SMP are two-stage stochastic programming (TSP) and chance-constrained programming (CCP). The SMP does not simplify the complexity of the programming problem but allow the effects of uncertainties as well as the relationships between uncertain inputs and resulting solutions to be reflected. The FMP is a mathematical programming model where the flexibility of the target values of the objective, the elasticity of the constraints, the parameters in either the objective or the constraints are quantified as fuzzy numbers. Two main types of FMP are fuzzy flexible programming (FFP) and fuzzy robust programming (FRP). The IMP can handle the optimization model where all or part of the parameters are expressed as interval numbers (i.e. a number with an unknown distribution between fixed lower and upper bounds). The two-step algorithm and the best worst case analysis represent two mainstream algorithms that are computationally efficient in obtaining interval solutions for an IMP model. It is much convenient to combine the IMP with other inexact programming methods to develop a hybrid method to tackle the inexact optimization problems under multiple uncertainties.

This paper presents a review on application of inexact programming methods to waste management under uncertainty. The selected work in this area will be grouped into eight sub-sections based on the mainly employed programming framework. The sub-sections include: two-stage stochastic programming, chance-constrained programming, fuzzy flexible programming, fuzzy robust programming, interval-parameter programming, inexact mixed-integer programming, inexact multiple-objective programming, and inexact nonlinear programming. Not only current status abut also future directions will be discussed as well.

\section{Review}

\section{Two-stage stochastic programming}

When the effects of random events on the decisionmaking process are a concern, the decision variables, costs and processes can be divided into two sets or belong to two stages, which is so-called two-stage stochastic programming (TSP) or programming with recourse (Sahinidis 2004). The first-stage sets are those to be decided before the random event occurs, which represent the target plan under various policy scenarios. In comparison, the second-stage ones are corresponding to all possibilities of the random event, which can be treated as corrective actions (recourses) against any infeasibility after actual random events have happened. The objective function is usually to minimize the sum of both the first-stage costs for the initial decisions and the expected value of the second-stage costs for the future recourse actions. To simply the calculation, the random variables approximate to a set of discrete values so that the TSP problem can be transformed to a linear programming model. The main disadvantages of TSP include the following aspects. The TSP cannot be applied when the quality of uncertain information is not satisfactory enough to be presented as random variables. For large-scale TSP problems in many real-world cases, the interactions among multiple random parameters and decision variables might lead to serious complexities. Compared with CCP, the TSP can hardly account for the violating risk of uncertain system constraints. Compared with FMP, the TSP has difficulties in tackling uncertainties in fuzzy membership functions.

The TSP framework has been applied to various environmental management problems. Especially in the area of waste management (Table 1), Maqsood and Huang (2003) for the first time developed a two-stage intervalstochastic programming (TISP) model and applied it to solid-waste management under uncertainty (wastegeneration rates are random variables). Following this study, Maqsood et al. (2004) proposed an inexact twostage mixed integer linear programming (ITMILP) model through integrating mixed integer, two-stage stochastic and interval-parameter programming approaches within a general optimization framework and applied ITMILP to the planning of regional solid waste management systems under uncertainty. Li et al. (2006a) presented an interval fuzzy two-stage stochastic mixed-integer linear programming (IFTSIP) method for planning waste-management systems under uncertainty through incorporating twostage stochastic, fuzzy flexible, mixed integer, and intervalparameter programming approaches within a general optimization framework. Li et al. (2006b) presented an interval-parameter two-stage stochastic mixed integer programming (ISMILP) technique for waste management under uncertainty, which is a hybrid of interval-parameter programming, TSP, and mixed integer linear programming methods. Li and Huang (2006) applied the ISMILP to supporting long-term waste management activities in Regina, which can help tackle the dynamic, interactive and uncertain characteristics of waste management, support adjustment or justification of the existing patterns for 
Table 1 Applications of two-stage stochastic programming to waste management

\begin{tabular}{|c|c|c|c|c|c|}
\hline TSP & IMP & MIP & CCP & FMP & Reference \\
\hline TSP & ILP & & & & (Maqsood and Huang 2003) \\
\hline TSP & ILP & MIP & & & (Li et al. 2008e; Li and Huang 2006; Li et al. 2006b; Maqsood et al. 2004) \\
\hline TSP & ILP & MIP & & FLP & (Li et al. 2006a) \\
\hline TSP & ILP & MIP & $\mathrm{ICP}$ & & (Li et al. 2006c; Su et al. 2009) \\
\hline TSP & ILP & MIP & $\mathrm{ICP}$ & FLP & (Li et al. 2008c; Li et al. 2009a) \\
\hline TSP & ILP & & & FLP & (Li et al. 2008d) \\
\hline TSP & $\| \mathrm{ILP}$ & & $\mathrm{ICP}$ & & Li and Huang (2007) \\
\hline TSP & ILP & & & FQP & (Li and Huang, 2007) \\
\hline TSP & IQP & & & & (Li et al. 2008a) \\
\hline TSP & IQP & & $\mathrm{ICP}$ & & (Sun et al. 2010a) \\
\hline TSP & ILP & MIP & & FRP & (Li et al. 2008b) \\
\hline TSP & $\mathrm{ILP}$ & MIP & & FCCP & (Guo and Huang 2009) \\
\hline
\end{tabular}

waste flow allocation, address long-term planning for cost-effective waste diversion and landfill prolongation, and analyze different policies regarding waste generation and management under different scenarios. $\mathrm{Li}$ et al. (2006c) proposed an internal-parameter two-stage chance-constrained mixed integer linear programming (ITCILP) method for municipal solid waste management problems, which can directly handle uncertainties in internals and probability density distributions, assess the satisfying reliability (or the violating risk) of various constraints, and analyze various policy scenarios associated with different levels of economic penalties when violating the promised policy targets.

Recently, Li and Huang (2007) constructed an inexact two-stage chance-constrained linear programming (ITCLP) method for planning waste management systems, which is derived by incorporating the techniques of TSP, chance-constrained programming, and interval-parameter programming. Li and Huang (2007a) developed a fuzzy two-stage quadratic programming (FTSQP) method for waste-management, which incorporates both fuzzy quadratic programming and TSP within a general optimization framework to quantify uncertainties expressed as probability-density and fuzzy-membership functions. Li et al. (2008c) developed an interval fuzzy two-stage chanceconstrained linear programming (IFTCP) method for longterm petroleum waste management planning, where uncertainties presented as intervals, fuzzy sets, and probability distributions can be effectively incorporated and the trade-off between system cost and system-failure risk can be analyzed thoroughly. Li et al. (2008e) applied an integrated two-stage optimization model (ITOM) to municipal solid waste management in Regina, which can support adjustment of the existing waste-management practices and identification of desired policies regarding waste generation and management. Li et al. (2008d) proposed an interval-fuzzy two-stage stochastic linear programming (IFTP) method for waste allocation, which integrates TSP, interval-parameter programming, and fuzzy linear programming within a framework. Li et al. (2008b) constructed a two-stage fuzzy robust integer programming (TFRIP) method, through integration of TSP, fuzzy robust programming, and a mixed integer linear programming, which facilitates dynamic analysis of capacity-expansion planning for waste management facilities within a multistage context and specifies the possibilistic information through dimensional enlargement of the original fuzzy constraints.

More recently, Guo and Huang (2009) constructed inexact fuzzy chance-constrained two-stage mixed-integer linear programming (IFCTIP) for supporting long-term planning of waste-management systems in Regina under uncertainties expressed as multiple uncertainties of intervals and dual probability distributions, which facilitates dynamic analysis for facility-expansion planning and waste-flow allocation within a multi-facility, multi-period, multi-level, and multi-option context. Li et al. (2009a) developed an interval-fuzzy two-stage chance-constrained integer programming (IFTCIP) method, based on integration of TSP, fuzzy linear programming, chance-constrained programming, interval-parameter programming, and mixed integer linear programming. The IFTCIP has advantages in reflecting uncertainties expressed as probability distributions, fuzzy sets, and discrete intervals, investigating policy scenarios associated with different levels of economic penalties once promised policy targets are violated, assessing risks of violating system constraints under various significance levels, and capacity-expansion analysis. Su et al. (2009) developed interval-parameter two-stage chanceconstraint mixed integer linear programming (ITCMILP) for supporting long-term planning of solid waste management in Foshan, China, based on integration of intervalparameter, two-stage, mixed integer, and chance-constraint programming methods into a general framework. In the 
ITCMILP model, three scenarios are examined to cover combinations of various system conditions and waste management policies. Sun et al. (2010a) proposed an inexact chance-constrained quadratic solid waste management (ICQSWM) model, which integrates two-stage stochastic, chance-constrained, and interval-parameter quadratic programming together.

\section{Chance-constrained programming}

When the uncertainties in the constraints' right-handside parameters are quantified as random variables, the constraints are not required to be totally satisfied. In this case, the chance-constrained programming (CCP) or probabilistic programming method can be used to handle the reliability of satisfying system constraints or violating system risks. The main disadvantages of CCP include the following aspects. Compared with FMP and IMP, it is usually difficult for CCP to tackle independent uncertainties of parameters in the constraints' left-hand-side and the objective function; compared with the TSP, the CCP is not designed for analyzing various policy scenarios associated with different economic penalties when the expected targets are violated; due to the data availability, when available information is not of high quality enough for establishing probability distribution functions for the right-hand-side parameters, the CCP may not be applicable.

Actually, the foregoing CCP is usually referred to as the individual probabilistic constrained programming (ICP). As one main type of CCP, the ICP only requires each constraint to be satisfied at a probability level. In other words, the relationships between the individual probabilities for the constraints are not reflected in an ICP model, which may result in inefficient performance in maintaining prescribed overall system reliability. In practice, the decision-makers sometimes may require the reliability levels to be imposed on the entire management system rather than on each constraint separately. Thus, as the other main type of CCP, joint-probabilistic constrained programming (JCP) is capable of dealing with the limitations. In a typical JCP model, all of uncertain constraints required being satisfied at a joint probability level, which increases robustness in controlling overall system risk during the optimization process.

The CCP method has wide applications to various environmental management problems. Some representative studies on applications of the CCP method to waste management are listed in Table 2. Huang et al. (2001b) developed an integrated fuzzy-stochastic linear programming model and applied it to municipal solid waste management, which integrates CCP, fuzzy linear programming, interval-parameter programming and mixed-integer linear programming within a general framework. Cai et al. (2007) developed a mixed interval parameter

\begin{tabular}{|c|c|c|c|c|c|c|}
\hline CCP & IMP & SMP & SIP & MIP & FMP & Reference \\
\hline $\mathrm{ICP}$ & ILP & & & MIP & FLP & (Huang et al. 2001b) \\
\hline ICP & ILP & & & & FRP & (Cai et al. 2007) \\
\hline ICP & ILP & & SIP & MIP & & (Guo et al. 2008a) \\
\hline ICP & ILP & TSP & SIP & MIP & & (Guo et al. 2008b) \\
\hline$I C P$ & ILP & SRP & & & & (Xu et al. 2009) \\
\hline ICP & ILP & & SIP & & & (Guo et al. 2009) \\
\hline JCP & DLP & & & MIP & & (Liu et al. 2009a) \\
\hline LICP & RBILP & & & & & (Cheng et al. 2009) \\
\hline $\mathrm{ICP}$ & ILP & & & & SI-FMP & (Tan et al. 2010a) \\
\hline$I C P$ & ILP & & & MIP & & (Su et al. 2010; Xi et al. 2010) \\
\hline LCP & ILP & & & & & (Sun et al. 2013) \\
\hline
\end{tabular}

fuzzy-stochastic robust programming (MIFSRP) model and applied it to solid waste management, based on CCP, interval-parameter programming, and fuzzy robust programming. In the MIFSRP model, fuzziness and randomness for the lower and upper bounds of interval parameters can be effectively reflected. Guo et al. (2008b) developed an interval-parameter two-stage stochastic semiinfinite programming (ITSSIP) method, which integrates CCP, two-stage stochastic programming, interval programming, and semi-infinite programming within a general optimization framework. The ITSSIP model can help generate optimal solutions under different waste-generation rates and multiple constraint-violation probabilities and tackle variations of the functional interval parameters with time. Guo et al. (2008a) conducted an inexact stochastic mixed integer linear semi-infinite programming (ISMISIP) model for municipal solid waste management, which incorporates $\mathrm{CCP}$, integer programming, interval parameter programming, and semi-infinite programming within a general waste management problem. The ISMISIP model can simultaneously quantify coefficients expressed as probability distribution functions, intervals and functional intervals without generating more complicated intermediate models during the solving process.

More recently, Xu et al. (2009) proposed hybrid stochastic robust chance-constraint programming (SRCCP) for supporting municipal solid waste management, which couples stochastic robust programming with CCP. In the SRCCP, the trade-offs among expected value of the objective function, variation in the value of the objective function and risk of violating constraints can be examined. Guo et al., (2009) developed an interval-parameter fuzzystochastic semi-infinite mixed-integer linear programming (IFSSIP) method for waste management, which integrates fuzzy programming, chance-constrained programming, integer programming, interval-parameter programming, and semi-infinite programming within an optimization 
framework. In the IFSSIP method, both dynamic features of interval-function conditions over the planning horizon, and stable ranges of solutions under fuzzy satisfaction degrees and different constraint-violating probabilities can be handled. Guo et al. (2008a) advanced an inexact stochastic mixed integer linear semi-infinite programming (ISMISIP) model for solid waste management, which incorporates stochastic programming, integer programming, and interval-parameter programming and semi-infinite programming within a general framework. The ISMISIP model can simultaneously tackle a waste management problem with coefficients expressed as probability distribution functions (capacities of the landfill, WTE and composting facilities), intervals and functional intervals, without requiring more complicated intermediate models.

Liu et al. (2009a) proposed a dual interval probabilistic integer programming (DIPIP) model for long-term waste management under uncertainty, which integrates joint probabilistic programming, dual interval programming, and mixed-integer linear programming. The DIPIP allows generating reasonable facility expansion schemes under uncertainties expressed as probability distributions as well as single and dual intervals. Cheng et al. (2009) proposed a random-boundary-interval linear programming (RBILP) method and applied it to municipal solid waste management under dual uncertainties, where random boundary intervals (intervals with random lower and upper bounds) in both left-hand-side and right-hand-side constraints can be handled. Sun et al. (2013) developed an inexact joint-probabilistic left-hand-side chance-constrained programming (IJLCP) method and applied it to a solid waste management problem under dual uncertainties. A nonequivalent but sufficient linearization form of IJLCP was proposed and proved in a straightforward manner for solving this type of problem.

In addition, Tan et al. (2010a) developed a superiorityinferiority-based inexact fuzzy-stochastic chanceconstrained programming (SI-IFSCCP) approach for supporting long-term waste management, where multiple uncertainties expressed as intervals, possibilistic and probabilistic distributions, two-layer randomness (two levels of system-violation risk), and various subjective judgments of multiple stakeholders with different interests and preferences, can be directly quantified. Xi et al. (2010) proposed an inexact chance-constrained mixed-integer linear programming (ICMILP) model for long-term solid waste management in Beijing, China, based on integration of the interval-parameter, mixed-integer, and CCP methods. Three waste management scenarios under lowest, medium, and highest diversion rates in Beijing were designed and evaluated through a fuzzy MCDA model. $\mathrm{Su}$ et al. (2010) developed an inexact chance-constraint mixed integer linear programming (ICMILP) model for supporting waste management in Foshan, China. The
ICMILP model can tackle uncertainties presented as intervals and probabilities, facilitate long-term capacity planning, and formulate policies regarding waste generation, collection, transportation and treatment.

\section{Fuzzy flexible programming}

When the flexibility in the constraints and fuzziness exist in the objective function, fuzzy constraints and fuzzy goals are introduced as fuzzy sets to conventional programming models. Thus, the elasticity of the constraints and the flexibility of the target values in the objective function can be quantified. Usually, the constraints' right-hand sides (available resource) and the target objective function values are presented as vaguer information. Through introduction of a fuzzy decision variable, not only the highest membership degree in the objective function but also a satisfactory degree for each constrained resource can be quantified as fuzzy membership functions and solved simultaneously. This type of FMP is the so-called fuzzy flexible programming (FFP). The main disadvantages of FFP include the following aspects. Compared with SMP, the membership functions of both the fuzzy objective and the constraints in the FFP should be determined subjectively by the decision makers. Compared with FRP, the FFP cannot handle the fuzziness of parameters in constraints' left-hand-sides.

Table 3 lists some representative studies on applications of the FFP framework to waste management. Huang et al. (1993) developed an interval fuzzy linear programming for waste management under uncertainty, which combines interval-parameter programming with FFP to tackle uncertainties in both the model coefficients and stipulations. Huang et al. (1994b) proposed an interval fuzzy quadratic programming (IFQP) approach for optimization analysis of waste management problems under uncertainty, which integrates interval-parameter programming, fuzzy linear programming and fuzzy quadratic programming within a general optimization framework. The IFQP

\begin{tabular}{|c|c|c|c|c|}
\hline FMP & IMP & MIP & Other methods & Reference \\
\hline FFP & $\mathrm{ILP}$ & & & $\begin{array}{l}\text { (Huang et al. 1993; } \\
\text { Srivastava and Nema 2011) }\end{array}$ \\
\hline FFP & IQP & & & (Huang et al. 1994b) \\
\hline FFP & ILP & MIP & MOP & (Chang et al. 1997b) \\
\hline FFP & ILP & & & (Huang et al. 2001b) \\
\hline FFP & ILP & & VA & (Huang et al. 2002; Li et al. 2009b) \\
\hline FFP & ILP & & MSP & (Li et al. 2009c) \\
\hline FFP & ILP & MIP & SIP & (He et al. 2008b) \\
\hline FFP & ILP & & SIP & (Huang et al. 2008) \\
\hline FFP & ILP & MIP & $\mathrm{BIP}$ & (He et al. 2009) \\
\hline
\end{tabular}


uses multiple control variables for the related fuzzy constraints instead of a single variable for all constraints to incorporate the independent properties of the stipulation uncertainties. Chang et al. (1997b) developed a fuzzy interval multi-objective mixed integer programming (FIMOMIP) model to evaluate sustainable management strategies for solid waste management in a metropolitan region. The application of FIMOMIP demonstrates that not only management cost/benefit but also environmental impacts (air pollution, traffic flow limitation, and leachate and noise impacts) can be minimized under uncertainties quantified by either membership functions or interval numbers. Huang et al. (2001b) proposed a fuzzy-stochastic linear programming model and applied it to municipal solid waste management. The model incorporates FFP, interval-parameter programming, CCP, and mixed-integer linear programming, which has advantages in uncertainty reflection, data availability, and computational requirement. Huang et al. (2002) proposed a violation analysis-based interval-parameter fuzzy integer programming (IPFIP) model for regional waste management, where the model's decision space can be expanded through introduction of violation variables to relax critical constraints. The solution of IPFIP provides considerable information related to facility expansion and waste flow allocation under given levels of tolerable violation for system constraints, which allows for in-depth analyses of tradeoffs between environmental and economic objectives as well as those between system optimality and reliability.

More recently, He et al. (2008b) proposed a fuzzy inexact mixed-integer semi-infinite programming (FIMISIP) method for waste management planning, which allows uncertainties expressed as fuzzy, interval, and functional interval numbers to be directly communicated into the problem. The FIMISIP model can address dynamic complexity through introduction of functional-interval parameters and provide a set of flexible waste-management schemes to the decision makers. Huang et al. (2008) developed a fuzzy interval semi-infinite programming model for waste management, which can allow for the existence of tolerance intervals for each of the constraints and address the possible effects of energy prices on the identified waste management policies. He et al. (2009) developed a flexible interval mixed-integer bi-infinite programming (FIMIBIP) method, which can allow parameters in the objective and constraints to be functional intervals, support diverting solid waste flow as well as sizing, timing and siting the facilities' expansion, reflect the level of constraints satisfaction, and quantify fluctuation of gas and energy prices. Li et al. (2009b) developed a constraintsoftened interval-fuzzy linear programming (CS-IFLP) method for violation analysis of waste management systems, which can deal with uncertainties presented in terms of fuzzy sets and intervals, allow fuzzy relaxation levels for system constraints, and help to analyze tradeoffs among economic objectives, satisfaction degrees, and constraint-violation risk. Li et al. (2009c) developed inexact fuzzy-stochastic constraint-softened programming for waste management through incorporation of multistage stochastic programming (MSP), ILP, and FFP. Srivastava and Nema (2011) proposed a fuzzy flexible programming model for selection of the treatment and disposal facilities, optimum capacity planning and waste allocation under uncertainty and applied it to waste management in Delhi, India.

\section{Fuzzy robust programming}

When the fuzziness in parameters is quantified as fuzzy sets, the uncertain parameters are represented as possibility distributions. The concept of alpha-level set is introduced to transform the fuzzy membership functions to fuzzy intervals so that ambiguous coefficients can be defuzzified. In other words, the decision space is delimited through dimensional enlargement of the original fuzzy constraints so that the fuzzy problem is converted to a corresponding deterministic one. This type of FMP is the so-called fuzzy robust programming (FRP) method. It should be noted that the FRP, as a type of possibilistic programming, is different from robust optimization (RO) or robust stochastic programming (Ben-Tal and Nemirovski 2002; Sahinidis 2004; Xu et al. 2010; Bertsimas et al. 2011).

In FRP, both left- and right-hand-side coefficients represented by possibilistic distributions in the constraints can be effectively handled. As for the main disadvantage of FRP, a large number of additional constraints and variables would be generated through the alpha-cut solution algorithm, which usually brings about complicated and time-consuming computation processes and may cause that no feasible solutions can be found. Compared with FFP, the FRP can hardly tackle the elasticity of the constraints and the flexibility of the target values in the objective function.

Table 4 lists the applications of the FRP to waste management. Nie et al. (2007) for the first time introduced FRP to the filed of waste management. He proposed

Table $\mathbf{4}$ Application of fuzzy robust programming to
waste management
\begin{tabular}{llll}
\hline FMP & IMP & Other Methods & Reference \\
\hline FRP & ILP & & (Nie et al. 2007; Li et al. 2010) \\
FRP & ILP & ICP & (Cai et al. 2007) \\
FRP & ILP & MIP & (Li et al. 2008b) \\
FRP & ILP & DP & (Nie et al. 2009) \\
FRP & ILP & MOP & (Zhang et al. 2010a) \\
FRP & ILP & FCCP & (Zhang and Huang 2010) \\
FRP & ILP & infinite alpha-Cuts & (Wang et al. 2011) \\
\hline
\end{tabular}


an interval-parameter fuzzy-robust programming (IFRP) model and applied it to the planning of solid waste management systems under uncertainty, where the concept of fuzzy boundary interval was introduced to handle fuzzy information for the lower and upper bounds of interval parameters. Following this study, Li et al. (2008b) developed a two-stage fuzzy robust integer programming (TFRIP) method to plan waste management systems under uncertainty, which integrates FRP, two-stage stochastic programming, and mixed integer linear programming. The TFRIP method can delimit fuzzy decision space through dimensional enlargement of the original fuzzy constraints, which provides desired plans for both waste-flow-allocation and capacity-expansion with a minimized system cost and maximized system feasibility. Nie et al. (2009) conducted an interval fuzzy robust dynamic programming (IFRDP) model for planning of waste-flow allocation and facility expansion, which combines fuzzy robust programming, interval-parameter programming, and dynamic programming within a framework. The IFRDP model allows fuzzy boundary intervals to be effectively communicated into dynamic programming and interval-parameter fuzzy robust programming to be embedded, which enhances robustness of the optimization process and the final solutions.

More recently, Li et al. (2010) introduced interval-fuzzy possibilistic programming (IFPP) to solving solid waste management problems under uncertainties expressed as interval values and fuzzy sets, which can help analyze tradeoffs among system cost, possibility degrees, and constraint-violation risk. Zhang et al. (2010a) proposed a fuzzy-robust stochastic multi-objective programming (FRSMOP) model for petroleum waste management, which integrates fuzzy-robust linear programming, stochastic linear programming, and multi-objective programming to generate a certain number of non-inferior solutions to reflect the decision-makers' preferences and subjectivity. The FRSMOP model can minimize system cost and waste flows directly to landfill simultaneously. Zhang and Huang (2010) developed fuzzy robust credibility-constrained programming (FRCCP) and applied it to planning for waste management systems, which couples fuzzy robust programming with credibility-based chance-constrained programming. To solve the FRCCP model, fuzzy credibility constraints are transformed to the crisp equivalents at credibility levels while the ordinary fuzzy constraints are replaced by the deterministic constraints at alpha-cut levels. Wang et al. (2011) developed interval-valued fuzzy linear programming with infinite alpha-cuts (IVFLP-I) and applied it to municipal solid waste management under uncertainty expressed as intervals and interval-valued fuzzy sets. The IVFLP-I model can deal with all fuzzy information through delimiting infinite alpha-cut levels to the interval-valued fuzzy membership function so as to help analyze tradeoffs between system costs and constraint-violation risks thoroughly.

\section{Interval-parameter programming}

When the quality of available data is insufficient for creating probability density distributions or fuzzy membership functions, the upper and lower bounds (intervals) of uncertain parameters can usually be easily obtained. Thus, based on the interval-number theory, an intervalparameter mathematical programming (IMP) model can be developed where all or part of the parameters are expressed as interval numbers (i.e. a number with an unknown distribution between fixed lower and upper bounds). The two-step algorithm and the best worst case analysis represent two mainstream algorithms that are computationally efficient in obtaining interval solutions for an IMP model (Rosenberg 2009; Fan and Huang 2012).

The advantages of the IMP would include the following aspects. The interval information can be directly communicated into the optimization process and the resulting solution. The solution algorithms do not generate more complicated intermediate models but require relatively low computational effort. Compared with SMP and FMP, the interval information for parameters in the IMP is more convenient to obtain than their distributional function or membership information, which is particularly meaningful for real-world applications. Thus it is convenient to incorporate the concepts of IMP and other inexact programming methods within a general framework to tackle more complicated complex or hybrid uncertainties (Table 5).

\begin{tabular}{lll}
$\begin{array}{l}\text { Table } \mathbf{5} \text { Applications of interval-parameter programming } \\
\text { to waste } \text { management }\end{array}$ \\
\begin{tabular}{lll} 
IMP & $\begin{array}{l}\text { Other } \text { methods } \\
\text { or features }\end{array}$ & Reference \\
\hline ILP & Two-step algorithm & (Huang et al. 1992) \\
ILP & FLP & (Huang et al. 1993) \\
ILP & DP & (Huang et al. 1994a) \\
ILP & Rough-interval & (Lu et al. 2008) \\
ILP & Dual-interval & (Liu et al. 2009b) \\
ILP & Radius interval & (Tan et al. 2010b) \\
ILP & Possibilistic interval numbers & (Zhang et al. 2010b) \\
ILP & IFQP & (Sun et al. 2010b) \\
ILP & FLP, IFQP & (Sun et al. 2011) \\
ILP & SRO & (Xu et al. 2010) \\
ILP & ThSM & (Cao and Huang 2011; \\
& & Huang and Cao 2011) \\
ILP & MRA & (Cui et al. 2011) \\
ILP & Reverse logistics & (Zhang et al. 2011) \\
ILP & RTSM & (Fan and Huang 2012) \\
\hline & &
\end{tabular} \\
\hline
\end{tabular}


However, the limitations of IMP include the following aspects. The IMP model may not have feasible solutions when the right-hand side parameters in constraints are highly uncertain. When the parameter quality is good enough to be expressed as distributional density functions or fuzzy membership functions, construction of an IMP model would lose the detailed information. Compared with TSP, the IMP can hardly quantify economic consequences of violating system constraints, which are essential for the related policy analyses (2003). The early applications of IMP to waste management were initialized by Dr. Huang. For the first time, Huang et al. (1992), introduced an interval linear programming (ILP) model to the area of waste management. The ILP model is applied to a hypothetical problem of waste flow allocation planning within a municipal solid waste management system, which allows interval uncertainties in the model inputs to be communicated into the optimization process and the output solutions reflecting the inherent uncertainties can be obtained. Following this seed study, Huang et al. (1993) proposed interval-fuzzy linear programming for optimization analysis under uncertainty, which couples interval linear programming with fuzzy flexible linear programming within a framework. Huang et al. (1994a) developed an interval dynamic programming (GDP) method for waste management, which couples interval linear programming with dynamic programming.

Recently, Lu et al. (2008) proposed a greenhouse gas (GHG) mitigation-induced rough-interval programming model for waste management under dual uncertainties, which integrated the concepts of rough-interval and GHG mitigation within a general interval-parameter programming framework. The model provided sustainable strategies to optimize waste allocation, mitigate GHG emissions, and control environmental pollution, which can analyze complicated interrelationships among solid waste management, climate-change impacts, and pollution control. Liu et al. (2009b) developed a dualinterval parameter linear programming (DILP) model and applied it to the planning of municipal solid waste management, which introduced the concept of dual interval (an interval-boundary interval) to the existing interval-parameter linear programming framework. The DILP model can generate decision alternatives through analysis of the single- and dual-interval solutions according to projected applicable conditions. Tan et al. (2010b) developed a radial-interval linear programming (RILP) approach for supporting waste management under uncertainty, which introduced the concept of fluctuation radius (uncertain information at the bounds of interval parameters) to the conventional interval-parameter linear programming framework. The RILP approach can provide a series of interval solutions under varied protection levels and help analyze the interactions among protection level, violation risk, and system cost under various projected system conditions as well as tolerance levels that decisionmakers will pay and risk. Zhang et al. (2010b) proposed a hybrid interval-parameter possibilistic programming (IPP) approach and applied it to municipal solid waste management under dual uncertainties, which introduced the concept of possibilistic interval numbers (lower and upper bounds of interval parameters have possibility distributions) to the objective function of interval-parameter programming.

More recently, Sun et al. (2010b) developed a fuzzyqueue-based interval linear programming (FQ-ILP) model for long-term municipal solid waste management planning, through introducing fuzzy queue (FQ) model into an ILP framework. The FQ-ILP model can help analyze policy scenarios associated with fuzzy arrival rates, fuzzy service rates, fuzzy waiting time, and different waiting and operation costs. Xu et al. (2010) proposed a stochastic robust interval linear programming model (IPRO) for supporting municipal solid waste management under uncertainty, which couples stochastic robust optimization with interval linear programming to analyze trade-offs among expected costs, cost variability, and risk of violating relax constraints. The IPRO model can help decision makers to identify desired waste management policies under various environmental, economic, system-feasibility and system-reliability constraints. Sun et al. (2011) developed an inexact fuzzy-queue programming (IFQP) model for solid waste management under uncertainty, which integrates FQ model, interval-parameter programming, and fuzzy flexible programming. The IFQP model can help analyze tradeoffs among system cost, satisfaction degrees, and environmental constraints considering the influence of FQ on decision-making problems.

Especially, Cao and Huang in 2011 developed a threestep method (ThSM) to guarantee that no infeasible solutions be included in the solutions of an interval-parameter programming model where all coefficients are assumed to obey normal or uniform distribution (Cao and Huang 2011; Huang and Cao 2011). The ThSM was applied to a municipal solid waste management problem under twelve scenarios according to the variations in concerns on objective function (aggressive, conservative, or neutral), the attitude to the constraints (optimistic or pessimistic), and the preferred types of constricting ratios (consistent or varied). The ThSM can generate a number of feasible schemes under twelve scenarios, which allows decision makers to further adjust the obtained solutions and identify a desired one based on their experiences, economic situations, social and cultural conditions. Cui et al. (2011) developed an interval-based regret-analysis (IBRA) model for supporting long-term planning of solid waste management activities in Changchun, China, which incorporates interval parameter programming, minimax-regret analysis, 
and mixed integer-programming. The IBRA model can help analyze economic consequences under different system costs and system-failure risk levels without assuming probabilistic distributions for random variables. Zhang et al. (2011) applied interval-parameter programming to solving a reverse logistics model for municipal solid waste management systems (IRWM), where waste managers, suppliers, industries and distributors were involved in strategic planning and operational execution. To solve the IRWM, a piecewise interval programming model was introduced to dealing with the minimization functions in both objectives and constraints. Fan and Huang (2012) developed a robust two-step method (RTSM) to solve interval-parameter linear programming through incorporating additional constraints into solution procedures. Compared with the TSM, the RTSM can provide a larger solution space and avoid absolute violation of certain constraints so that loss of decision-related information is prevented.

\section{Inexact mixed-integer programming}

Inexact mixed-integer programming (IMIP) is categorized as three main types: fuzzy MIP, stochastic MIP and interval MIP. The fuzziness and randomness may exist not in integer decision variables but in real-number ones; on the contrary, integer decision variables can be interval numbers, which can represent the set of limited discrete options. Compared with real-time decision variables, integer decision variables are able to determine the discrete options, such as facility expansion options. However, if the number of integer decision variables in a programming model is too large, the searching complexity of solution algorithm would increase exponentially. Among inexact mixed-integer programming, interval-parameter mixedbinary programming would be the main type which has been applied to the area of solid waste management (Table 6).

\begin{tabular}{|c|c|c|c|}
\hline IMIP & FLP & Other methods & Reference \\
\hline IMIP & & & (Huang et al. 1995b; 1997; 2005) \\
\hline IMIP & FLP & & (Huang et al. 1995a) \\
\hline IMIP & FLP & TSP & (Huang et al. 2001a) \\
\hline IMIP & FLP & VA & (Huang et al. 2002) \\
\hline IMIP & & MCDA & (Cheng et al. 2003) \\
\hline IMIP & & GT & $\begin{array}{l}\text { (Davila and Chang 2005; } \\
\text { Davila et al. 2005) }\end{array}$ \\
\hline IMIP & & GHG & (Lu et al. 2009) \\
\hline IMIP & & MR & $\begin{array}{l}\text { (Li and Huang 2009a, } \\
\text { Li and Huang 2009b) }\end{array}$ \\
\hline IMIP & FLP & SIP, FCCP & (Guo and Huang 2010) \\
\hline
\end{tabular}

Huang et al. (1995b) proposed an interval integer programming (IIP) method for facility expansion planning within a regional solid waste management system, which integrated interval-parameter programming and mixed integer linear programming within an optimization framework. The binary variable solutions indicated different development alternatives within a multi-period, multifacility and multi-scale context. Huang et al. (1995a) developed an interval fuzzy integer programming method and applied it to facility expansion/utilization planning within a regional solid waste management system. The model integrated interval-parameter programming, fuzzy flexible programming and mixed integer linear programming within an optimization framework. Huang et al. (1997) applied interval integer programming to the capacity planning of an integrated waste management system in the Regional Municipality of Hamilton-Wentworth (RMHW), Ontario, Canada. Huang et al. (2001a) developed an inexact fuzzy-stochastic mixed integer linear programming (IFSMILP) model and applied it to an integrated solid waste management system in the City of Regina. Huang et al. (2002) developed a violation-analysisbased interval-parameter fuzzy integer programming (VA-IPFIP) model and applied it to planning of regional solid waste management systems. In the model, the given levels of tolerable violation for several critical constraints are explicitly expressed. The model can help analyze tradeoffs between environmental and economic objectives as well as those between system optimality and reliability within a facility expansion and waste flow allocation problem.

Cheng et al. (2003) developed an integrated approach which combined multi-criteria decision analysis (MCDA) with an interval mixed integer linear programming model to support landfill site selection and waste flow allocation in Regina. The MCDA methods to evaluate the landfill site alternatives include simple weighted addition, weighted product, co-operative game theory, TOPSIS, and complementary ELECTRE. Davila and Chang (2005) developed interval mixed integer programming for optimal shipping patterns and capacity planning of material recovery facilities in San Antonio, Texas. In the model, waste generation, incidence of recyclables in the waste stream, routing distances, recycling participation, and other planning components are quantified as intervals. The constraints consist of mass balance, capacity limitation, recycling limitation, scale economy, conditionality, and relevant screening restrictions. Davila et al. (2005) proposed an interval integer programming (IIP) model to generate a strategic plan for optimal solid waste patterns with minimized net costs for cities in the Lower Rio Grande Valley (LRGV) region in South Texas and developed an IIP-based twotiered games analysis for evaluating optimal pricing strategies for tipping fees available to the most significant regional landfills. Huang et al. (2005) developed inexact 
mixed integer linear programming for long-term planning of an integrated solid waste management (ISWM) system in Regina. The model can provide solutions of siting, timing, and sizing for new and expanded waste management facilities in relation to a variety of waste-diversion targets.

Lu et al. (2009) developed an inexact dynamic optimization model (IDOM), which combined the concept of greenhouse gas (GHG) emission mitigation with a mixed-integer linear programming model. The model can generate waste-flow patterns with a minimized system cost and GHG-emission amount, which successfully quantify the impacts of waste management on GHG emissions. $\mathrm{Li}$ and Huang, (2009a, b) developed an inexact minimax regret integer programming (IMMRIP) method for the long-term planning of municipal solid waste management in Regina. The IMMRIP model integrated minimax regret analysis, interval-parameter programming, and mixedinteger linear programming within a framework, which can help analyze decisions of system-capacity expansion and/or development within a multi-facility and multiperiod context. Guo and Huang (2010) developed an interval-parameter semi-infinite fuzzy-chance-constrained mixed-integer linear programming (ISIFCIP) approach for supporting long-term waste-management planning in Regina. The model integrated mixed-integer linear programming, interval-parameter programming, semi-infinite programming and fuzzy-chance-constrained programming within a general framework, which can tackle multiple uncertainties expressed as intervals, functional intervals (dual uncertainties), random variables, fuzzy sets, and their combinations (fuzzy-interval admissible probability).

\section{Inexact multiple-objective programming}

Compromises among multiple conflicting objectives need to be made in real-world cases under uncertainty. The less important objectives would be converted to constraints implicitly while the most important one would be kept in conventional single-objective programming. In comparison, inexact multiple-objective programming (IMOP) can be developed to handle different objectives more explicitly. To solve the IMOP, evaluation of importance to different objectives would be the core problem. When different types of uncertainties exist, the IMOP can be classified as: fuzzy MOP, stochastic MOP and interval MOP. Since the solution algorithm of the FFP can transform multiple objective functions to equivalent constraints, it makes the FFP straightforward to deal with importance of multiple objectives. The random variables and interval numbers usually exist in the parameters of different objectives and constraints, which would rarely affect the algorithm to handle weights among objectives in the corresponding IMOP. The IMOP has been applied to waste management problems (Table 7 ).
Chang and Lu (1996 and 1997) developed a fuzzy multiobjective mixed integer programming model and applied it to long-term solid waste management planning in Kaohsiung, Taiwan. The model considered socioeconomic and environmental impacts simultaneously and allowed fuzzy environmental resources to be incorporated into the optimization processes. Chang and Wang (1997) developed fuzzy goal programming for the optimal planning of solid waste management systems in a metropolitan region. In the model, four objectives including economic costs, noise control, air pollution control, and traffic congestion limitations were considered. Chang and Chen (1997) developed an interval fuzzy goal programming model and applied it to waste management under uncertainties. The model's results demonstrated how the interval-parameter values and fuzzy messages in goals can be quantified within the framework, which helped interpret the complexity from both system nature and human aspiration. Chang et a1. (1997b) developed a fuzzy interval multiobjective mixed integer programming (FIMOMIP) model and applied it to municipal solid waste planning. The model minimized overall management cost under the effects of various environmental considerations (air pollution, traffic flow limitation, and leachate and noise impacts).

Recently, Ahluwalia and Nema (2006) presented an inexact integer linear goal programming model based on material flow analysis and Monte Carlo simulation for computer waste management. The economy, health and environmental risks associated with various computer waste management activities were also evaluated. He et al. (2008a) developed an interval full-infinite programming (IFIP) method through introduction of functional intervals into an optimization framework and applied it to waste management planning with infinite objectives and constraints under uncertainty. The IFIP can help address the complex relationships between inexact parameters and their external impact factors within a multi-objective waste management framework. Chaerul et al. (2008) developed an inexact integer linear programming model based on Monte Carlo simulation and applied it to computer waste management planning in Delhi, India. The model can help address the environmental problems associated with exponentially growing quantities of computer waste. Ahluwalia and Nema (2011) developed a multi-time-step and multi-objective inexact decisionsupport model for computer waste management. The model can address multiple objectives of waste management cost, environmental risk, and health risk within a management framework for the optimum configuration of existing and proposed facilities.

\section{Inexact nonlinear programming}

The management problems are nonlinear in nature, which makes inexact nonlinear programming (INP) an 
Table 7 Application of inexact multiple-objective programming to waste management

\begin{tabular}{|c|c|c|c|c|c|c|}
\hline MOP & ILP & FLP & MIP & GP & Other methods & Reference \\
\hline MOP & & FLP, & MIP & & & (Chang and Lu 1996; Chang and Lu 1997) \\
\hline MOP & & FLP & & GP & & (Chang and Wang 1997) \\
\hline MOP & ILP & FLP & & GP & & (Chang and Chen 1997) \\
\hline MOP & $I L P$ & FLP & MIP & & & (Chang et al. 1997b) \\
\hline MOP & & & MIP & GP & MCS & (Ahluwalia and Nema 2006) \\
\hline MOP & ILP & FLP & & & SIP & (He et al. 2008a) \\
\hline MOP & & & MIP & & MCS & (Chaerul et al. 2008) \\
\hline MOP & & & & & DSS & (Ahluwalia and Nema 2011) \\
\hline
\end{tabular}

indispensable method. The INP can be classified as fuzzy nonlinear programming, stochastic nonlinear programming, and interval nonlinear programming. When both uncertainty and nonlinearity exist within an INP framework, mathematically proved algorithms, which convert the nonlinear forms to a corresponding linear one, need to be developed,. The transformed linear form can be equivalent, non-equivalent but sufficient, or approximated expressions of the original nonlinear equations. Thus, the finally obtained linear model under uncertainty can be further converted to deterministic linear models, which can be solved by conventional algorithms. Due to the limitation of solution algorithms, the current interval nonlinear programming applied to waste management usually focuses on relatively simple nonlinear expressions (Table 8).

Huang et al. (1994b) proposed an interval fuzzy quadratic programming (IFQP) approach and applied it to waste management, which integrated interval-parameter linear programming and fuzzy quadratic programming within a general optimization framework. The IFQP

Table 8 Application of inexact nonlinear programming to waste management

\begin{tabular}{lll}
\hline NP & Other methods or features & Reference \\
\hline FQP & ILP & (Huang et al. 1994b) \\
IQP & & (Huang and Baetz 1995) \\
INP & FGP & (Chang and Wang 1996) \\
INP & MIP & (Chang et al. 1997a) \\
INP & FLP, MOP, GA, MIP & (Chang and Wei 2000) \\
IQP & More Efficient Algorithm & (Chen and Huang 2001) \\
INP & Exponential Objective Function & (Wu et al. 2006) \\
IQP & TSP, FLP & (Li and Huang; 2007) \\
IQP & TSP & (Li et al. 2008a) \\
IQP & CCP & (Guo et al. 2008c) \\
IQP & FRP & (Sun et al. 2009) \\
IQP & TSP & (Guo and Huang 2011) \\
INP & Piecewise Linearization & (Sun et al. 2012) \\
\hline
\end{tabular}

model incorporated the independent properties of the stipulation uncertainties through induction of multiple control variables for each constraint. Huang and Baetz (1995) developed an interval-parameter quadratic programming (IQP) method and applied it to waste management, which combined interval-parameter linear programming with quadratic programming. Based on IQP, the effects of economies of scale on cost coefficients in the objective function can be quantified. Chang and Wang (1996) developed a nonlinear fuzzy goal programming approach for solving conflicting solid-waste management goals. The emphasis were put on complexity of composition, generation, and heat values of the waste streams, waste reduction and recycling requirements prior to incineration and emission control of trace organic compounds during incineration in the decision making. Chang et al. (1997a) developed nonlinear mixed integer programming to minimize total operational costs for a large-scale solid-waste collection, recycling, treatment, and disposal system.

Chang and Wei (2000) developed a genetic-algorithmaided fuzzy multi-objective nonlinear integer programming model to allocate the recycling drop-off stations with appropriate sizes in the solid waste collection network to maximize the recycling achievement with minimum expense in the city of Kaohsiung in Taiwan. Chen and Huang (2001) developed and proved a derivative algorithm for solving the inexact quadratic programming model (IQP) with much lower computational efforts, which is especially meaningful for the IQP's application to large-scale problems. Wu et al. (2006) proposed an interval nonlinear programming model with an exponential objective function and linear constraints, proved a satisfactory algorithm to solve the model, and applied them to the planning of waste management activities with economics-of-scale effects on system costs in the Hamilton-Wentworth Region of Ontario, Canada. Li and Huang (2007a) developed a fuzzy two-stage quadratic programming (FTSQP) method for waste-management. The FTSOQP improves upon the existing fuzzy linear programming methods through more effectively both minimizing the variation of satisfaction degrees among 
the objective and constraints, and tackling the trade-off between the system cost and the constraint-violation risk.

Li et al. (2008a) developed an inexact stochastic quadratic programming model to handle nonlinear cost objective functions reflecting the effects of economies of scale and applied it to a case of long-term waste-management planning. The model integrated interval-parameter programming, quadratic programming and two-stage stochastic programming within a general framework. Guo et al. (2008c) developed an interval stochastic quadratic programming method (ISQP) and applied it to a municipal solid waste management system with multiple disposal facilities and multiple cities within multiple periods, which integrated chance-constrained programming and IQP within a general framework. Sun et al. (2009) developed interval fuzzy robust nonlinear programming (IFRNLP) and applied it to municipal solid waste management. The IFRNLP can reflect system cost variations more effectively and generate more applicable solutions than other conventional methods. Guo and Huang (2011) developed an inexact fuzzy-stochastic quadratic programming (IFSQP) method to allocate waste to available facilities with minimized total expected system cost over the entire planning horizon. The constraints of IFSQP include relationships among decision variables, waste generation rates, waste diversion goals, and facility capacities. Sun et al. (2012) developed an inexact piecewise-linearization-based fuzzy flexible programming (IPFP) model to tackle nonlinear economies-of-scale effects in interval-parameter constraints for a representative waste management problem.

\section{Recommendations for future research}

Based on the literature review, the demanding areas for future research efforts would be recommended as follows:

(1) More work needs to be conducted on integration of a single inexact programming method with other programming methods to deal with multiple uncertainties and their interactions. Many parameters in waste management problems are subject to uncertainties presented as intervals, random variables, fuzzy sets, and their combinations (Sun and Huang 2010; Sun et al. 2012). These multiple uncertainties may be present in a single parameter simultaneously, exist in multiple parameters within a programming model, or interact with each other due to the inherent tradeoffs or the overall system risk. Such interactive and multiple uncertainties may lead to difficulties in identifying desired waste management plans.

(2) Greater attention should be paid to integration of inexact programming with other nonlinear programming to handle both uncertainties and nonlinearities. The waste management problem is nonlinear inherently and uncertain inevitably. Consideration of both uncertainties and nonlinearities would not only help the waste management programming models to approximate to the actual characteristics of real-world cases, but also make the solution of such models more complicated (Sun et al. 2013). Accordingly, development of more efficient algorithms would be desired to solve the proposed inexact nonlinear models (Sahinidis 2004; Zhou et al. 2008; Zhou et al. 2009).

(3) Research on integration of inexact programming with other modeling technologies would be a promising field. In the conventional inexact programming methods, most of the parameters are estimated by simple inference from historical data or prior experiences of decision makers. These parameters can hardly be more reasonably calculated without help of the simulation models (Beigl et al. 2008). In addition, the schemes generated by the inexact programming models are usually evaluated by the corresponding objective function values. Life cycle assessment (LCA) and multiple-criteria decision analyses (MCDA) would be desired to help decision makers to choose more practical and sustainable schemes (Morrissey and Browne 2004; Kaplan et al. 2009). Integration of these models within a user-friendly decision support system would be also helpful to enhance their applicability in real-world waste management problems.

(4) Applications of the developed methods to novel or real-world cases in waste management systems would be another challenge. Especially, certain types of wastes, such as electronic wastes, petroleum wastes, or hazardous wastes should be separately considered within a specific waste management system (Qin et al. 2009). Linkage of waste management with its environmental impacts (e.g. air pollutants and GHG emissions as well as leachate pollution) within an inexact optimization framework would need significant research efforts (Levis et al. 2013; Mavrotas et al. 2013). The waste management systems in different countries may have their unique characteristics (Guerrero et al. 2013; Laner et al. 2012; Levis et al. 2010), which are worthy of further investigation through application of the inexact programming methods.

\section{Conclusions}

The literature review highlights the development and applications of inexact programming methods to waste management under uncertainty, which have become a popular research area. As a promising systems-analysis tool, the inexact programming methods can help to analyze the tradeoff among different components within the system, to quantify various types of multiple uncertainties in parameters and their relations, and to generate schemes for planning long-term waste management. The development trend of optimization methods for waste management would include: integration of a single inexact programming method with other programming methods to deal with multiple uncertainties and their interactions, integration of inexact programming with other nonlinear 
programming to handle both uncertainties and nonlinearities with the help of more efficient algorithms, integration of inexact programming with other modeling techniques (e.g. LCA, MCDA, and waste flow simulation) to support sustainable waste management, linkage of waste management with its environmental impacts (e.g. air pollutants and GHG emissions as well as leachate pollution) within an inexact optimization framework, and applications of the developed methods to novel (e.g. specific types of wastes) or real-world waste management cases in different countries.

\begin{abstract}
Abbreviations
CCP: Chance-constrained programming; DP: Dynamic programming; FFP: Fuzzy flexible programming; FMP: Fuzzy mathematical programming; FQP: Fuzzy quadratic programming; FQ: Fuzzy queue; FRP: Fuzzy robust programming; FCCP: Fuzzy chance-constrained programming; GHG: Green-house gas; GT: Game theory; ILP: Interval-parameter programming; IMP: Interval-parameter mathematical programming; IQP: Interval-parameter quadratic programming; ICP: Individual chance-constrained programming; LCA: Life cycle assessment; MCDA: Multi-criteria decision analysis; MCS: Monte Carlo simulation; MIP: Mixed integer programming; MR: Mini-max regret; MSP: Multistage stochastic programming; RBILP: Random-boundary-interval linear programming; RO: Robust optimization; RTSM: Robust two-step method; SI-FMP: Superiority-inferiority-based fuzzy mathematical programming; SIP: Semi-infinite programming; SMP: Stochastic mathematical programming; SRP: Stochastic robust programming; TSP: Two-stage stochastic programming; ThSM: Three step methods; VA: Violation analysis.
\end{abstract}

\section{Competing interests}

The authors declare that they have no competing interests.

\section{Authors' contributions}

The work was carried out in collaboration among all authors. SW defined the research topic, compared the inexact programming methods, and drafted most of the manuscript; AC collected the references and drafted the tables; LG analyzed the advantages and disadvantage of each method; LY drafted recommendations for future research and revised the manuscript. All authors have approved the final manuscript.

\section{Acknowledgement}

This research was supported by the Natural Sciences Foundation of China (71303017), the Program for Innovative Research Team (IRT1127), the MOE Key Project Program (311013), the Natural Science and Engineering Research Council of Canada, and the Major Project Program of the Natural Sciences Foundation (51190095). The authors deeply appreciate the editors and the anonymous reviewers for their insightful comments and suggestions.

\section{Author details \\ 'Institute for Energy, Environment and Sustainable Communities, University of Regina, Regina, SK S4S 7H9, Canada. ${ }^{2}$ MOE Key Laboratory for Transportation Complex Systems Theory and Technology, School of Traffic and Transportation, Beijing Jiaotong University, Beijing 100044, China.}

Received: 24 December 2013 Accepted: 16 April 2014

Published: 20 June 2014

\section{References}

Abichou T, Clark J, Tan S, Chanton J, Hater G, Green R, Goldsmith D, Barlaz MA, Swan N (2010) Uncertainties associated with the use of optical remote sensing technique to estimate surface emissions in landfill applications. J Air Waste Ma 60:460-470

Ahluwalia PK, Nema AK (2006) Multi-objective reverse logistics model for integrated computer waste management. Waste Manage Res 24:514-527

Ahluwalia PK, Nema AK (2011) Capacity planning for electronic waste management facilities under uncertainty: multi-objective multi-time-step model development. Waste Manage Res 29:694-709
Belien J, De Boeck L, Van Ackere J (2014) Municipal solid waste collection and management problems: a literature review. Transport Sci 48:78-102

Beigl P, Lebersorger S, Salhofer S (2008) Modelling municipal solid waste generation: a review. Waste Manage 28:200-214

Ben-Tal A, Nemirovski A (2002) Robust optimization - methodology and applications. Math Program 92:453-480

Bertsimas D, Brown DB, Caramanis C (2011) Theory and applications of robust optimization. SIAM Rev 53:464-501

Cai YP, Huang GH, Nie XH, Li YP, Tan Q (2007) Municipal solid waste management under uncertainty: a mixed interval parameter fuzzy-stochastic robust programming approach. Environ Eng Sci 24:338-352

Cao M, Huang G (2011) Scenario-based methods for interval linear programming problems. J Environ Inform 17:65-74

Chaerul M, Tanaka M, Shekdar AV (2008) Resolving complexities in healthcare waste management: a goal programming approach. Waste Manage Res 26:217-232

Chang NB, Chang YH, Chen YL (1997a) Cost-effective and equitable workload operation in solid- waste management systems. J Environ Eng-ASCE 123:178-190

Chang NB, Chen YL (1997) Optimal balance between recycling and waste-to-energy prographs by grey fuzzy nonlinear goal programming.1. Theory. J Environ Sci Heal A 32:633-647

Chang NB, Chen YL, Wang SF (1997b) A fuzzy interval multiobjective mixed integer programming approach for the optimal planning of solid waste management systems. Fuzzy Set Syst 89:35-60

Chang NB, Lu HY (1996) Long Term Planning Of Solid Waste Management Systems by Fuzzy Compromise Programming. Proceedings I, 7Th Iswa International Congress, pp I353-1360

Chang NB, Lu HY (1997) A new approach for long term planning of solid waste management systems using fuzzy global criterion. J Environ Sci Heal A 32:1025-1047

Chang NB, Wang SF (1996) Managerial fuzzy optimal planning for solid- waste management systems. J Environ Eng-ASCE 122:649-658

Chang NB, Wang SF (1997) A fuzzy goal programming approach for the optimal planning of metropolitan solid waste management systems. Eur J Oper Res 99:303-321

Chang NB, Wei YL (2000) Siting recycling drop-off stations in urban area by genetic algorithm-based fuzzy multiobjective nonlinear integer programming modeling. Fuzzy Set Syst 114:133-149

Chang NB, Pires A, Martinho G (2011) Empowering systems analysis for solid waste management: challenges, trends, and perspectives. Crit Rev Env Sci Tec 41:1449-1530

Chen MJ, Huang GH (2001) A derivative algorithm for inexact quadratic program - application to environmental decision-making under uncertainty. Eur J Oper Res 128:570-586

Cheng G, Huang G, Li Y, Cao M, Fan Y (2009) Planning of municipal solid waste management systems under dual uncertainties: a hybrid interval stochastic programming approach. Stoch Env Res Risk A 23:707-720

Cheng S, Chan CW, Huang GH (2003) An integrated multi-criteria decision analysis and inexact mixed integer linear programming approach for solid waste management. Eng Appl Artif Intel 16:543-554

Cui L, Chen L, Li Y, Huang G, Li W, Xie Y (2011) An interval-based regret-analysis method for identifying long-term municipal solid waste management policy under uncertainty. J Environ Manage 92:1484-1494

Davila E, Chang NB (2005) Sustainable pattern analysis of a publicly owned material recovery facility in a fast-growing urban setting under uncertainty. J Environ Manage 75:337-351

Davila E, Chang NB, Diwakaruni S (2005) Landfill space consumption dynamics in The Lower Rio Grande Valley by grey integer programming-based games. J Environ Manage 75:353-365

Fan YR, Huang GH (2012) A robust two-step method for solving interval linear programming problems within an environmental management context. J Environ Inform 19:1-9

Ghiani G, Lagana D, Manni E, Musmanno R, Vigo D (2014) Operations research in solid waste management: a survey of strategic and tactical issues. Comput Oper Res 44:22-32

Guerrero LA, Maas G, Hogland W (2013) Solid waste management challenges for cities in developing countries. Waste Manage 33:220-232

Guo P, Huang G (2009) Inexact fuzzy-stochastic mixed-integer programming approach for long-term planning of waste management - part a: methodology. J Environ Manage 91:461-470 
Guo P, Huang G (2011) Inexact fuzzy-stochastic quadratic programming approach for waste management under multiple uncertainties. Eng Optimiz 43:525-539

Guo P, Huang G, He L (2008a) ISMISIP: an inexact stochastic mixed integer linear semi-infinite programming approach for solid waste management and planning under uncertainty. Stoch Env Res Risk A 22:759-775

Guo P, Huang G, He L, Li H (2009) Interval-parameter fuzzy-stochastic semi-infinite mixed-integer linear programming for waste management under uncertainty. Environ Model Assess 14:521-537

Guo P, Huang G, He L, Sun B (2008b) ITSSIP: Interval-parameter two-stage stochastic semi-infinite programming for environmental management under uncertainty. Environ Model Softw 23:1422-1437

Guo P, Huang G, Li Y (2008c) Interval stochastic quadratic programming approach for municipal solid waste management. J Environ Eng Sci 7:569-579

Guo P, Huang GH (2010) Interval-parameter semi-infinite fuzzy-stochastic mixed-integer programming approach for environmental management under multiple uncertainties. Waste Manage 30:521-531

He L, Huang G, Tan Q, Liu Z (2008a) An interval full-infinite programming method to supporting environmental decision-making. Eng Optimiz 40:709-728

He L, Huang G, Zeng G, Lu H (2008b) Fuzzy inexact mixed-integer semi-infinite programming for municipal solid waste management planning. J Environ Eng-ASCE 134:572-581

He L, Huang GH, Lu HW (2009) Flexible interval mixed-integer bi-infinite programming for environmental systems management under uncertainty. J Environ Manage 90:1802-1813

Huang G, Cao M (2011) Analysis of solution methods for interval linear programming. J Environ Inform 17:54-64

Huang GH, Baetz BW (1995) Grey quadratic-programming and its application to municipal solid- waste management planning under uncertainty. Eng Optimiz 23:201-223

Huang GH, Baetz BW, Patry GG (1992) A gray linear-programming approach for municipal solid- waste management planning under uncertainty. Civil Eng Syst 9:319-335

Huang GH, Baetz BW, Patry GG (1993) A gray fuzzy linear-programming approach for municipal solid- waste management planning under uncertainty. Civil Eng Syst 10:123-146

Huang GH, Baetz BW, Patry GG (1994a) Grey dynamic-programming for wastemanagement planning under uncertainty. J Urban Plan D-ASCE 120:132-156

Huang GH, Baetz BW, Patry GG (1994b) Waste flow allocation planning through a grey fuzzy quadratic-programming approach. Civil Eng Syst 11:209-243

Huang GH, Baetz BW, Patry GG (1995a) Grey fuzzy integer programming - an application to regional waste management planning under uncertainty. Socio Econ Plan Sci 29:17-38

Huang GH, Baetz BW, Patry GG (1995b) Grey integer programming - an application to waste management planning under uncertainty. Eur J Oper Res 83:594-620

Huang GH, Baetz BW, Patry GG, Terluk V (1997) Capacity planning for an integrated waste management system under uncertainty: a North American case study. Waste Manage Res 15:523-546

Huang GH, Chi GF, Li YP (2005) Long-term planning of an integrated solid waste management system under uncertainty - I. Model development. Environ Eng Sci 22:823-834

Huang GH, Sae-Lim N, Chen Z, Liu L (2001a) Long-term planning of waste management system in the City of Regina - an integrated inexact optimization approach. Environ Model Assess 6:285-296

Huang GH, Sae-Lim N, Liu L, Chen Z (2001 b) An interval-parameter fuzzy-stochastic programming approach for municipal solid waste management and planning. Environ Model Assess 6:271-283

Huang GH, He L, Zeng GM, Hw L (2008) Identification of optimal urban solid waste flow schemes under impacts of energy prices. Environ Eng Sci 25:685-695

Huang YF, Baetz BW, Huang GH, Liu L (2002) Violation analysis for solid waste management systems: an interval fuzzy programming approach. J Environ Manage 65:431-446

Juul N, Munster M, Ravn H, Soderman ML (2013) Challenges when performing economic optimization of waste treatment: a review. Waste Manage 33:1918-1925

Kaplan PO, Ranjithan SR, Barlaz MA (2009) Use of life-cycle analysis to support solid waste management planning for delaware. Environ Sci Technol 43:1264-1270

Laner D, Crest M, Scharff H, Morris JWF, Barlaz MA (2012) A review of approaches for the long-term management of municipal solid waste landfills. Waste Manage 32:498-512
Levis JW, Barlaz MA, DeCarolis JF, Ranjithan SR (2013) A generalized multistage optimization modeling framework for life cycle assessment-based integrated solid waste management. Environ Model Softw 50:51-65

Levis JW, Barlaz MA, Themelis NJ, Ulloa P (2010) Assessment of the state of food waste treatment in the United States and Canada. Waste Manage 30:1486-1494

Li YP, Huang G, Nie SL, Huang YF (2006a) IFTSIP: interval fuzzy two-stage stochastic mixed-integer linear programming: a case study for environmental management and planning. Civ Eng Environ Syst 23:73-99

Li YP, Huang G, Nie SL, Nie XH, Maqsood I (2006b) An interval-parameter two-stage stochastic integer programming model for environmental systems planning under uncertainty. Eng Optimiz 38:461-483

Li YP, Huang G, Baetz BW (2006c) Environmental management under uncertainty - An internal-parameter two-stage chance-constrained mixed integer linear programming method. Environ Eng Sci 23:761-779

Li YP, Huang G (2006) An inexact two-stage mixed integer linear programming method for solid waste management in the City of Regina. J Environ Manage 81:188-209

Li YP, Huang G, Guo P, Nie SL (2010) Interval-fuzzy possibilistic mixed integer linear programming for environmental management under uncertainty. Int J Environ Pollut 42:107-124

Li YP, Huang G, Liu Y, Zhang Y, Nie SL (2008a) An inexact stochastic quadratic programming method for municipal solid waste management. Civ Eng Environ Syst 25:139-155

Li YP, Huang G, Nie XH, Nie SL (2008b) A two-stage fuzzy robust integer programming approach for capacity planning of environmental management systems. Eur J Oper Res 189:399-420

Li Y, Huang G, Qin X, Nie SL (2008c) IFTCP: an integrated method for petroleum waste management under uncertainty. Pet Sci Technol 26:912-936

Li Y, Huang G, Xiao H (2008d) Municipal solid waste management under uncertainty: an interval-fuzzy two-stage stochastic programming approach. J Environ Inform 12:96-104

Li Y, Huang G, Yang Z, Nie S (2008e) An integrated two-stage optimization model for the development of long-term waste-management strategies. Sci Total Environ 392:175-186

Li Y, Huang G, Yang Z, Nie S (2009a) IFTCIP: an integrated optimization model for environmental management under uncertainty. Environ Model Assess 14:315-332

Li YP, Huang GH (2007) Fuzzy two-stage quadratic programming for planning solid waste management under uncertainty. Int I Syst Sci 38:219-233

Li Y, Huang G, Yang Z, Nie S (2009b) A constraint-softened interval-fuzzy linear programming approach for environmental management under uncertainty. Environ Eng Sci 26:1335-1348

Li YP, Huang G, Yang Z, Chen X (2009c) Inexact fuzzy-stochastic constraintsoftened programming - A case study for waste management. Waste Manage 29:2165-2177

Li Y, Huang GH (2009a) Inexact minimax regret integer programming for long-term planning of municipal solid waste management - part A: methodology development. Environ Eng Sci 26:209-218

Li Y, Huang GH (2009b) Inexact minimax regret integer programming for long-term planning of municipal solid waste management - part B: application. Environ Eng Sci 26:219-234

Liu Z, Huang G, Liao R, He L (2009a) DIPIT: dual interval probabilistic integer programming for solid waste management. J Environ Inform 14:66-73

Liu Z, Huang G, Nie X, He L (2009b) Dual-Interval linear programming model and its application to solid waste management planning. Environ Eng Sci 26:1033-1045

Lu H, Huang G, He L, Zeng G (2009) An inexact dynamic optimization model for municipal solid waste management in association with greenhouse gas emission control. J Environ Manage 90:396-409

Lu H, Huang G, Liu Z, He L (2008) Greenhouse gas mitigation-induced rough-interval programming for municipal solid waste management. J Air Waste Ma 58:1546-1559

Maqsood I, Huang GH, Zeng GM (2004) An inexact two-stage mixed integer linear programming model for waste management under uncertainty. Civ Eng Environ Syst 21:187-206

Maqsood I, Huang GH (2003) A two-stage interval-stochastic programming model for waste management under uncertainty. J Air Waste Ma 53:540-552

Mavrotas G, Skoulaxinou S, Gakis N, Katsouros V, Georgopoulou E (2013) A multi-objective programming model for assessment the GHG emissions in MSW management. Waste Manage 33:1934-1949

Marshall RE, Farahbakhsh K (2013) Systems approaches to integrated solid waste management in developing countries. Waste Manage 33:988-1003 
Morrissey AJ, Browne J (2004) Waste management models and their application to sustainable waste management. Waste Manage 24:297-308

Nie X, Huang G, Li Y, Liu L (2007) IFRP: a hybrid interval-parameter fuzzy robust programming approach for waste management planning under uncertainty. J Environ Manage 84:1-11

Nie X, Huang GH, Li Y (2009) Capacity planning for waste management systems: an interval fuzzy robust dynamic programming approach. J Air Waste Ma 59:1317-1330

Pires A, Martinho G, Chang NB (2011) Solid waste management in European countries: a review of systems analysis techniques. J Environ Manage 92:1033-1050

Qin XS, Huang GH, He L (2009) Simulation and optimization technologies for petroleum waste management and remediation process control. J Environ Manage 90:54-76

Rosenberg DE (2009) Shades of grey: a critical review of grey-number optimization. Eng Optimiz 41:573-592

Sahinidis NV (2004) Optimization under uncertainty: state-of-the-art and opportunities. Comput Chem Eng 28:971-983

Singh A (2012) An overview of the optimization modelling applications. J Hydrol 466-467:167-182

Srivastava AK, Nema AK (2011) Fuzzy parametric programming model for integrated solid waste management under Uncertainty. J Environ Eng-ASCE 137:69-83

Su J, Huang G, Xi B, Qin X, Huo S, Jiang Y, Chen X (2010) Long-term panning of waste diversion under interval and probabilistic uncertainties. Resour Conserv Recy 54:449-461

Su J, Huang GH, Xi BD, Li YP, Qin XS, Huo SL, Jiang Y (2009) A hybrid inexact optimization approach for solid waste management in the city of Foshan, China. J Environ Manage 91:389-402

Sun H, Li Y, Huang G, Suo M (2011) An inexact fuzzy-queue programming model for environmental systems planning. Eng Appl Artif Intel 24:840-849

Sun W, Huang GH (2010) Inexact piecewise quadratic programming for waste flow allocation under uncertainty and nonlinearity. J Environ Inform 16:80-93

Sun W, Huang GH, Lv Y, Li G (2012) Waste management under multiple complexities: Inexact piecewise-linearization-based fuzzy flexible programming. Waste Manage 32:1244-1257

Sun W, Huang GH, Lv Y, Li G (2013) Inexact joint-probabilistic chance-constrained programming with left-hand-side randomness: an application to solid waste management. Eur J Oper Res 228(1):217-225

Sun Y, Huang G, Li Y, Xu Y, Cao M (2009) An interval fuzzy robust nonlinear program for the planning of municipal solid waste management systems under uncertainty. Eng Optimiz 41:989-1016

Sun Y, Huang GH, Li YP (2010a) ICQSWM: an inexact chance-constrained quadratic solid waste management model. Resour Conserv Recy 54:641-657

Sun Y, Li Y, Huang G (2010b) Development of a fuzzy-queue-based interval linear programming model for municipal solid waste management. Environ Eng Sci 27:451-468

Tan Q, Huang G, Cai Y (2010a) A superiority-inferiority-based inexact fuzzy stochastic programming approach for solid waste management under uncertainty. Environ Model Assess 15:381-396

Tan Q, Huang GH, Cai Y (2010b) Radial-interval linear programming for environmental management under varied protection levels. J Air Waste Ma 60:1078-1093

Verderame PM, Elia JA, Li J, Floudas CA (2010) Planning and scheduling under uncertainty: a review across multiple sectors. Ind Eng Chem Res 49:3993-4017

Wang S, Huang G, Lu H, Li Y (2011) An interval-valued fuzzy linear programming with infinite alpha-cuts method for environmental management under uncertainty. Stoch Env Res Risk A 25:211-222

Wu XY, Huang GH, Liu L, Li JB (2006) An interval nonlinear program for the planning of waste management systems with economies-of-scale effects - a case study for the region of Hamilton, Ontario, Canada. Eur J Oper Res 171:349-372

Xi B, Su J, Huang G, Qin X, Jiang Y, Huo S, Ji D, Yao B (2010) An integrated optimization approach and multi-criteria decision analysis for supporting the waste-management system of the City of Beijing, China. Eng Appl Artif Intel 23:620-631

Xu Y, Huang G, Qin X, Cao M (2009) SRCCP: A stochastic robust chance-constrained programming model for municipal solid waste management under uncertainty. Resour Conserv Recy 53:352-363

Xu Y, Huang G, Qin X, Cao M, Sun Y (2010) An interval-parameter stochastic robust optimization model for supporting municipal solid waste management under uncertainty. Waste Manage 30:316-327
Zeng Y, Cai YP, Huang GH, Dai J (2011) a review on optimization modeling of energy systems planning and GHG emission mitigation under uncertainty. Energies 4:1624-1656

Zhang X, Huang GH, Chan CW, Liu Z, Lin Q (2010a) A fuzzy-robust stochastic multiobjective programming approach for petroleum waste management planning. Appl Math Model 34:2778-2788

Zhang X, Huang GH, Nie X, Chen Y, Lin Q (2010b) Planning of municipal solid waste management under dual uncertainties. Waste Manage Res 28:673-684

Zhang YM, Huang GH, He L (2011) An inexact reverse logistics model for municipal solid waste management systems. J Environ Manage 92:522-530

Zhang Y, Hang G (2010) Fuzzy robust credibility-constrained programming for environmental management and planning. J Air Waste Ma 60:711-721

Zhou F, Guo HC, Chen GX, Huang GH (2008) Interval linear programming: a revisit. J Environ Inform 11:1-10

Zhou F, Huang GH, Chen GX, Guo HC (2009) Enhanced-interval linear programming. Eur J Oper Res 199:323-333

\section{doi:10.1186/s40068-014-0015-9}

Cite this article as: Sun et al:: Applications of inexact programming methods to waste management under uncertainty: current status and future directions. Environmental Systems Research 2014 3:15.

\section{Submit your manuscript to a SpringerOpen ${ }^{\circ}$ journal and benefit from:}

- Convenient online submission

- Rigorous peer review

- Immediate publication on acceptance

- Open access: articles freely available online

- High visibility within the field

- Retaining the copyright to your article

Submit your next manuscript at $>$ springeropen.com 